\title{
A Rare Hepatic Epithelioid Hemangioendothelioma in a Cirrhotic Liver
}

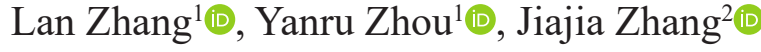 \\ ${ }^{1}$ Department of MRI, The First Affiliated Hospital of Henan University of Chinese Medicine, Zhengzhou, China \\ ${ }^{2}$ Department of Radiology, Gold Coast University Hospital, Queensland, Australia
}

To the Editor,

A 60 -year-old woman with a 20 years history of hepatitis B presented with 2 weeks of dull pain in the right upper quadrant and intermittent nausea. On admission, blood tests demonstrated serum alpha-fetoprotein $(8 \mu \mathrm{g} / \mathrm{L})$, carbohydrate antigen $125(10.3 \mathrm{U} / \mathrm{mL})$, carbohydrate antigen $199(9.9 \mathrm{U} / \mathrm{mL})$, and liver function were within the normal range. Abdominal magnetic resonance imaging (MRI) showed a solitary lesion as a multi-layered target appearance resembling a "maze" in Segment VIII of the liver. On diffusion-weighted image and T2-weighted images, the mass surprisingly showed 6 layers with alternate high and low signals (Figure 1A-B). The lesion did not demonstrate as many layers on T1-weighted image, however, a target appearance was still recognizable (Figure 1C). On Gadolinium ethoxybenzyldiethy-lenetriaminepentaacetic acid (Gd-EOB-DTPA)-enhanced MRI, the lesion showed progressive rim enhancement on the arterial and portal venous phase (Figure 1D-E) and appeared as a low signal target with a distinct hypointense core on the hepatobiliary phase (Figure 1F). The patient underwent laparoscopic lobectomy, and histopathology confirmed hepatic epithelioid hemangioendothelioma (HEHE). Microscopically, the tumor was composed of epithelioid and dendritic cells, which were positive for CD34, CD31, and CK-7 (Figure 2A-D).

Hepatic epithelioid angioendotheliomas are vascular endothelial cell tumors with low incidence rate and intermediate malignancy. ${ }^{1}$ It shows a slight predilection for females, with the average age at 30-50 years. ${ }^{1}$ Most patients have no history of hepatitis, and the clinical symptoms are not specific. The patients could be asymptomatic or present with nonspecific symptoms such as abdominal pain, fatigability, or weight loss. Hepatic epithelioid angioendotheliomas may present as a single or multifocal lesions, and cases of a single lesion account for less than $20 \%$ of HEHE. ${ }^{1}$
Characteristic imaging features of HEHE include "halo sign," "lollipop sign," "target sign," and capsular retraction. The common manifestation of "halo sign" consists of 3 layers of varying signals on multiple MRI sequences, which is known to be related to necrosis, prior hemorrhage, thrombosis, calcification, and myxoid stroma. ${ }^{2}$ Interestingly, our case showed a solitary lesion with as many as 6 layers, which is not the typical "halo sign" or "target sign." "Lollipop sign" is one of the specific imaging features, caused by tapering and termination of portal and hepatic vein and their branches when approaching the lesion. ${ }^{3}$ However, there is no convincing adjacent vessels forming "lollipop sign." Overall appearances are more like a "maze," which is a challenge for diagnosis. This maze-like manifestation actually makes hepatocellular carcinoma or metastasis as the main differentials being very unlikely, as metastasis typically has up to 3 layers with central necrosis, rim of peripheral active tumor, and outer layer of edema. Other differentials such as cholangiocarcinoma, atypical hemangioma, and angiosarcoma are also unlikely to show more than 3 layers.

The common finding of HEHE on the hepatobiliary phase is an internal enhancement surrounded by a low signal halo as "entrapmentlike" pattern. ${ }^{4}$ This "entrapment-like" pattern is nonspecific, which can also be observed in metastasis or cholangiocarcinoma. The lesion in our case shows a "core pattern" with a hypointense center on the hepatobiliary phase. Core pattern, regarded as a novel imaging feature of HEHE, is different from hepatocellular carcinoma, metastasis, or cholangiocarcinoma. ${ }^{5}$ Recognition of core pattern might further assist differential diagnosis.

Although the final diagnosis of HEHE requires pathological confirmation, being familiar with the imaging features and pathology-radiology correlation would be of great value for diagnosis. In conclusion, our case highlights that HEHE needs to be taken into consideration when a multi-layer appearing lesion is found in a cirrhotic liver.

\footnotetext{
Corresponding author: Lan Zhang, Department of MRI, The First Affiliated Hospital of Henan University of Chinese Medicine, Zhengzhou, China e-mail: 13837187787@163.com

Received: September 4, 2021 Accepted: October 8, 2021 •DOI: 10.5152/balkanmedj.2021.21902

Available at www.balkanmedicaljournal.org
}

ORCID iDs of the authors: L.Z. 0000-0002-7891-3964; Y.Z. 0000-0002-1311-4184; J.Z. 0000-0002-7615-2137.

Cite this article as:

Zhang L, Zhou Y, Zhang J. A rare hepatic epithelioid hemangioendothelioma in a cirrhotic liver. Balkan Med J. 2021; 38(6):394-396.

Copyright@Author(s)-Available online at http://balkanmedicaljournal.org/ 

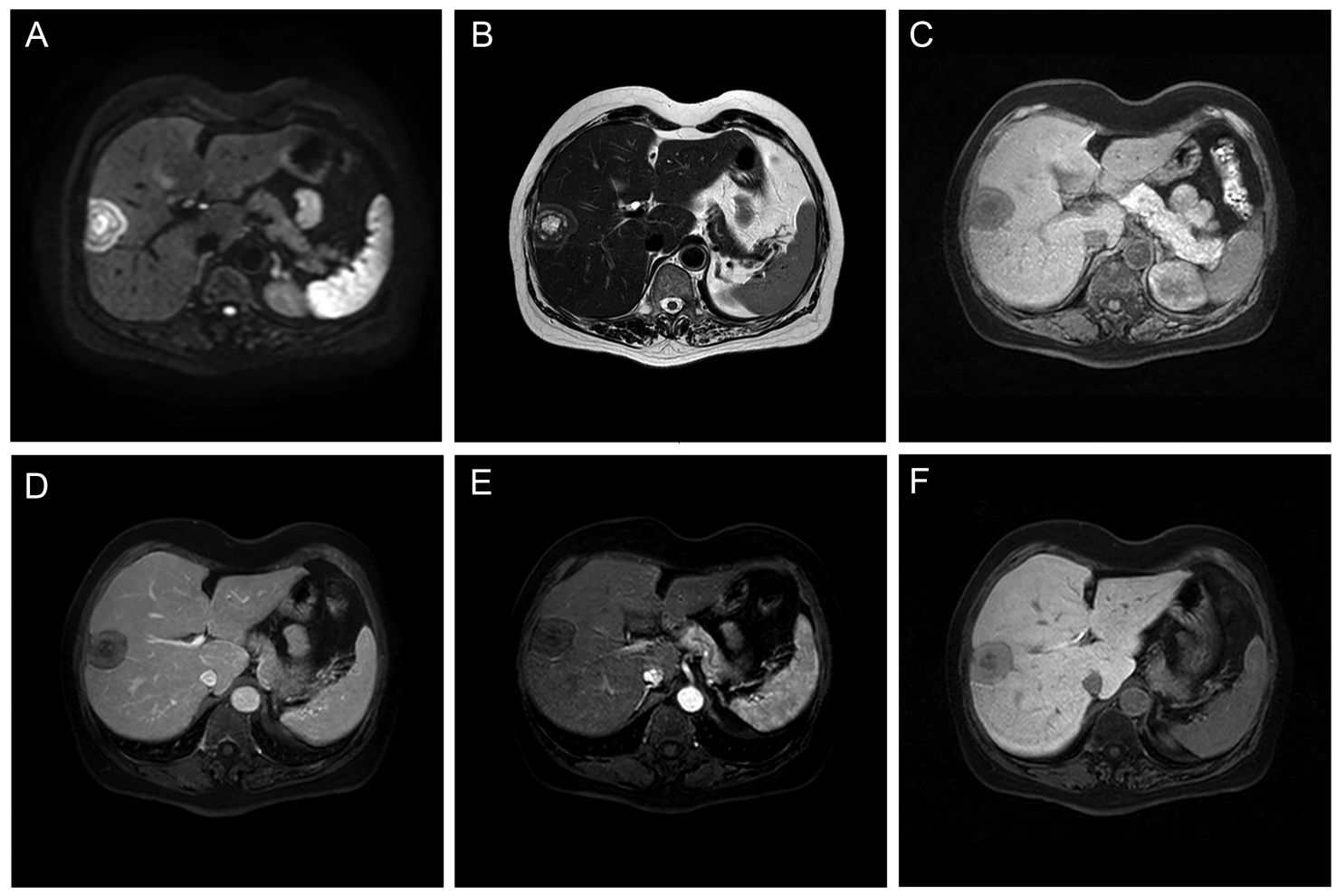

FIG. 1. A-F. (A) The tumor shows a 6-layered heterogeneous signal intensity on DWI and (B) T2WI, and (C) a target appearance on T1WI with progressive rim enhancement on the (D) arterial and portal venous phase (E). and as a low signal target with a hypointense core on the hepatobiliary phase (F). DWI, diffusionweighted image; T1W1, 1T-weighted images; T2W2, T2-weighted images.
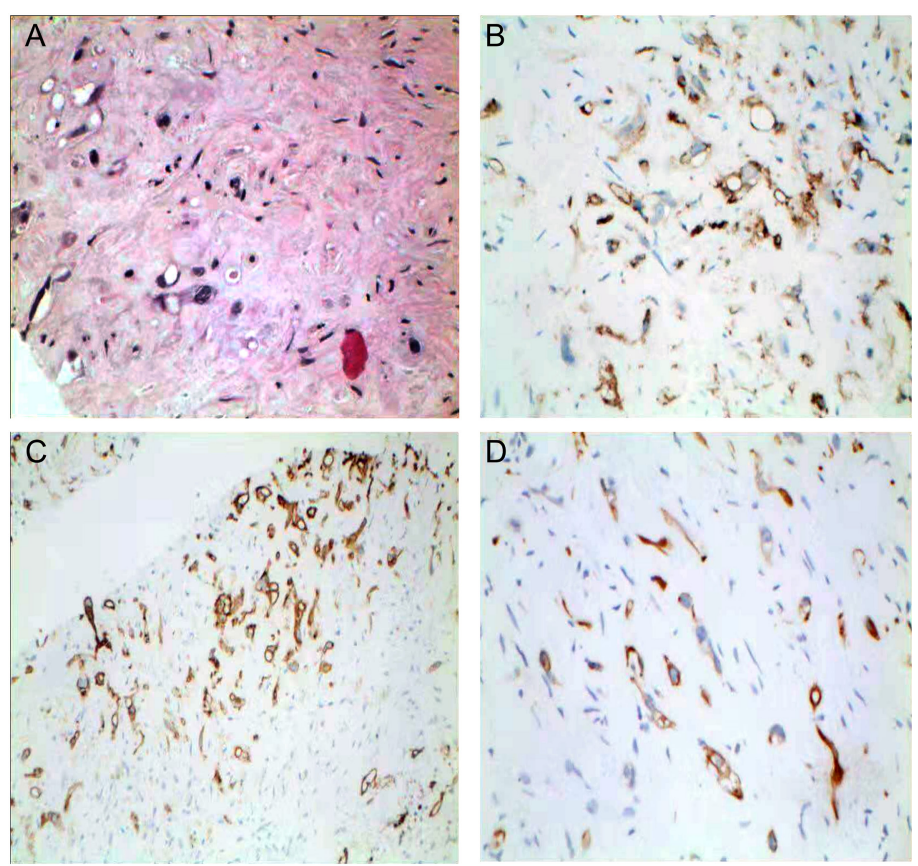

FIG. 2. A-D. (A) Epithelioid and dendritic cells forming primitive vascular structures with a myxoid matrix (H\&E, $\times 400)$. Positive staining for (B) CD34, (C) CD31, and (D) CK-7 ( $\times 200)$. 
Patient Consent for Publication: An informed consent was obtained from the patient for the publication.

Author Contributions: Concept - L.Z., J.Z.; Design - L.Z., J.Z.; Analysis and/or Interpretation - L.Z., Y.Z. J.Z.; Writing - L.Z.

Conflict of Interest: The authors have no conflict of interest to declare.

Funding: This study was funded by Henan Scientific and Technological Project (212102310732).

\section{REFERENCES}

1. Mehrabi A, Kashfi A, Fonouni H, et al. Primary malignant hepatic epithelioid hemangioendothelioma: a comprehensive review of the literature with emphasis on the surgical therapy. Cancer. 2006;107(9):2108-2121. [CrossRef]

2. Epelboym Y, Engelkemier DR, Thomas-Chausse F, et al. Imaging findings in epithelioid hemangioendothelioma. Clin Imaging. 2019;58:59-65. [CrossRef]

3. Alomari AI. The lollipop sign: a new cross-sectional sign of hepatic epithelioid hemangioendothelioma. Eur J Radiol. 2006;59(3):460-464. [CrossRef]

4. Paolantonio P, Laghi A, Vanzulli A, et al. MRI of hepatic epithelioid hemangioendothelioma (HEH). J Magn Reson Imaging. 2014;40(3):552-558. [CrossRef]

5. Lee JH, Jeong WK, Kim YK, et al. Magnetic resonance findings of hepatic epithelioid hemangioendothelioma: emphasis on hepatobiliary phase using Gd-EOB-DTPA. Abdom Radiol. 2017;42(9):2261-2271. [CrossRef] 